Revista de Matemática: Teoría y Aplicaciones 2004 11(2) : 35-53

CIMPA - UCR - CCSS ISSN: 1409-2433

\title{
DEFINICIÓN DE SEMIPRODUCTOS ESCALARES ÚTILES EN ANÁLISIS DE DATOS
}

\author{
JAVIER TREJOS-ZELAYA*
}

Recibido/Received: 11 Sept 2003

\begin{abstract}
Se desarrolla la teoría necesaria para realizar el Análisis de Datos en presencia de semiproductos escalares, extendiendo los conceptos clásicos de productos escalares usualmente empleados. Para ello, retomamos las definiciones algebraicas básicas de las formas bilineales no degeneradas y vamos desarrollando todas las herramientas algebraicas necesarias. Se estudian los operadores más importantes en el espacio de individuos, como el operados $V M$ y el operador $M V$. También se estudia el caso del semiproducto escalar de pesos en el espacio de variables, que en el caso de pesos nulos corresponde a la introducción de individuos suplementarios. Finalmente, llegamos a los conceptos usuales del Análisis en Componentes Principales.
\end{abstract}

Palabras clave: semiproductos escalares, formas bilineales no degeneradas, semimétricas, operador de proyección ortogonal, análisis en componentes principales.

\begin{abstract}
We develop the theory necessary for Data Analysis with inner semiproducts, extending tha classical concepts of inner products usually employed. For this, we use the basic algebraic definitions of non degenerated bilinear forms and develop all the algebraic tools needed. We study the most important operators on the individual space, such as the $V M$ and the $M V$ operators. We also study the case of the inner semiproduct of weights in the variable space, which corresponds to the introduction of supplementary individuals in the case of null weights. Finally, we arrive to the usual concepts of Principal Component Analysis.
\end{abstract}

Keywords: inner semiproducts, non degenerated bilinear forms, semimetrics, orthogonal projection operator, principal component analysis.

Mathematics Subject Classification: 11E39, 15A09, 15A63, 62H25

${ }^{*}$ CIMPA, Escuela de Matemática, Universidad de Costa Rica, 2060 San José, Costa Rica. E-Mail: jtrejos@cariari.ucr.ac.cr 


\section{Introducción}

Em algunos métodos de Análisis de Datos, se encuentran dificultades para definir una distancia en el espacio de los individuos. Este es el caso en los análisis canónicos cuando las variables están muy correlacionadas, pues la matriz de covarianzas es entonces numéricamente no invertible, y por ello es difícil calcular la matriz de una distancia de Mahalanobis. Hemos encontrado dificultades similares para definir una distancia entre conjunciones de modalidades esplicativas $[12,14,13]$. Hemos por lo tanto estudiado, en una primera parte, el uso de semiproductos escalares en Análisis de Datos. En una segunda parte, estudiamos algunas propiedades - en este contexto de semiproductos escalaresde dos operadores útiles en Análisis de Datos. Finalmente, abordamos la definición de algunos conceptos clásicos del Análisis de Datos, como los relacionados con la dispersión de una nube y el Análisis en Componentes Principales. Hacemos notar que estos temas los hemos abordado en otras publicaciones de circulación restringida [11, 14, 13].

\section{Algunos resultados sobre los semiproductos escalares}

Sean $L$ un espacio vectorial de dimensión finita, $L^{*}$ su espacio dual, $H$ un subespacio de $L$ y $T$ una forma bilineal simétrica sobre $L$. Se denota con la misma letra $T$ la aplicación lineal de $L$ en $L^{*}$ associada a $T$. $T_{/ H}$ es la restricción de la aplicación $T$ a $H$.

Proposición 1 Si $T$ es una forma bilineal simétrica sobre L, T la aplicación lineal asociada y $H$ un subespacio de $L$, se tiene:

a) Si $T$ es no degenerada sobre $H$ entonces la restricción $T_{/ H}$ de $T$ en $H$ es inyectiva.

b) Si $T_{/ H}$ es inyectiva y si $\operatorname{rang} T=\operatorname{dim} H$ entonces $T$ es no degenerada sobre $H$.

c) Si $T_{/ H}$ es inyectiva y si $T$ es positiva entonces $T$ es no degenerada sobre $H$.

\section{Demostración:}

a) Sea $x \in \operatorname{ker} T_{/ H} . \forall y \in H$ se tiene $T(x, y)=T(x)(y)=T_{/ H}(x)(y)=0$. Por hipótesis, $T$ es no degenerada sobre $H$, de donde se tiene $\{z \in H / \forall y \in H, T(y, z)=0\}=\{0\}$, luego $x=0 \mathrm{y}_{/ H}$ es inyectiva.

b) Se tiene $\operatorname{Im} T_{/ H} \subset \operatorname{Im} T$. Como por hipótesis $T_{/ H}$ es inyectiva $\mathrm{y}$ $\operatorname{rang} T=\operatorname{dim} H$, se tiene $\operatorname{dim}\left(\operatorname{Im} T_{/ H}\right)=\operatorname{dim} H=\operatorname{rang} T$. Luego $\operatorname{Im} T_{/ H}=\operatorname{Im} T$, es decir $\{T(y) / y \in H\}=\{T(y) / y \in L\}$.

Sea $x \in H$ tal que $\forall y \in H, T(x, y)=T(y)(x)=0$.

Se deduce que $\forall y \in L, T(x)(y)=T(y)(x)=0$, es decir, $T(x)=0$ y entonces $x \in H \cap \operatorname{ker} T=\operatorname{ker} T_{/ H}$.

Como $T_{/ H}$ es inyectiva se tiene $x=0$ y $T$ es por tanto no degenerada sobre $H$.

c) Sea $x \in H$ tal que $\forall y \in H, T(x, y)=0$. Como $T$ es positiva por hipótesis, se tiene $\operatorname{ker} T=\{z \in L / T(z, z)=0\}$, de donde $x \in H \cap \operatorname{ker} T=\operatorname{ker} T_{/ H}$.

En vista de que $T_{/ H}$ es inyectiva se tiene $x=0$ y luego $T$ es no degenerada sobre $H$. 
Nótese que la hipótesis rang $T=\operatorname{dim} H$ del inciso (b) de la proposición anterior podría ser reemplazada por $\operatorname{rang} T_{/ H}=\operatorname{rang} T$.

Proposición 2 Si $H$ y $G$ son dos subespacios vectoriales de L, con ortogonales respectivos $H^{\circ}$ y $G^{\circ}$ en $L^{*}$, se tiene:

$$
L=H \oplus G \Leftrightarrow L^{*}=H^{\circ} \oplus G^{\circ} .
$$

Demostración: Supńgase que $L=H \oplus G$. Sea $f \in H^{\circ} \cap G^{\circ}, \forall z \in L \quad z=x+y$ donde $x \in H$ y $y \in G, f(z)=f(x+y)=f(x)+f(y)=0$, de donde se deduce que $f=0$ y $H^{\circ} \cap G^{\circ}=\{0\}$. Por consiguiente $L^{*}=H^{\circ} \oplus G^{\circ}$ puesto que $\operatorname{dim} H^{\circ}=\operatorname{dim} L-\operatorname{dim} H$, $\operatorname{dim} G^{\circ}=\operatorname{dim} L-\operatorname{dim} G$ y $\operatorname{dim} L=\operatorname{dim} L^{*}$.

El recíproco es immediato al identificar $L^{* *}$ y $L$, puesto que $H^{\circ \circ}=H, G^{\circ \circ}=G$.

Denotando $\tilde{H}$ (respectivamente $\tilde{G}$ ) el espacio vectorial canónicamente isomorfo a $H$ (resp. $G$ ), si $L=H \oplus G$ entonces $G^{\circ}$ y $H^{\circ}$ son respectivamente isomorfos a los espacios duales $\tilde{H}^{*}$ y $\tilde{G}^{*}$.

En efecto, se pueden definir dos aplicaciones $\phi$ y $\psi$ sobre $L^{*}, \phi: L^{*} \rightarrow \tilde{H}^{*}$ y $\psi: L^{*} \rightarrow \tilde{G}^{*}$, cuyas restricciones respectivas a los subespacios $G^{\circ}$ y $H^{\circ}$ son tales que:

- $\forall y^{\circ} \in G^{\circ}$ y $\forall \tilde{x} \in \tilde{H}, \phi / G^{\circ}\left(y^{\circ}\right)(\tilde{x})=\phi\left(y^{\circ}\right)(\tilde{x})=y^{\circ}(x)$, donde $x$ es la imagen en $H$ de $\tilde{x}$ por la inyección canónica de $\tilde{H}$ en $H$.

- $\forall x^{\circ} \in H^{\circ}$ y $\forall \tilde{y} \in \tilde{G}, \psi_{/ H^{\circ}}\left(x^{\circ}\right)(\tilde{y})=\psi\left(x^{\circ}\right)(\tilde{y})=x^{\circ}(y)$, donde $y$ es la imagen en $G$ de $\tilde{y}$ por la inyección canónica de $\tilde{G}$ en $G$.

$\phi_{/ G^{\circ}} \mathrm{y} \psi_{/ H^{\circ}}$ son evidentemente lineales. Además, son biyectivas. En efecto, se tiene para $\phi / G^{\circ}:$

- $\operatorname{dim} \tilde{H}^{*}=\operatorname{dim} \tilde{H}=\operatorname{dim} H=\operatorname{dim} G^{\circ}$;

- Sea $y^{\circ} \in \operatorname{ker} \phi_{/ G^{\circ}}=G^{\circ} \cap \operatorname{ker} \phi, \forall x \in H \quad y^{\circ}(x)=\phi_{/ G^{\circ}}\left(y^{\circ}\right)(\tilde{x})=0$, luego $y^{\circ} \in H^{\circ}$.

Como $H^{\circ} \cap G^{\circ}=\{0\}$, se tiene $y^{\circ}=0$ y entonces $\operatorname{ker} \phi_{/ G^{\circ}}=\{0\}$.

Es análogo para $\psi_{/ H^{\circ}}$.

Para la descomposición $L=H \oplus G$, es entonces natural llamar $H^{\circ}$ (resp. $G^{\circ}$ ) al subespacio dual de $G$ (resp. $H$ ) y denotarlo $G^{*}$ (resp. $H^{*}$ ). Diremos además que $L=H \oplus G$ y $L^{*}=H^{*} \oplus G^{*}$ son dos descomposiciones duales de $L$ y $L^{*}$.

\section{Observaciones:}

1) Si $\left\{h_{1}, \ldots, h_{p}, g_{1}, \ldots, g_{q}\right\}$ es una base de $L$ tal que $\forall i \quad h_{i} \in H$ y $\forall j \quad g_{j} \in G$, cuya base dual en $L^{*}$ es $\left\{h_{1}^{*}, \ldots, h_{p}^{*}, g_{1}^{*}, \ldots, g_{q}^{*}\right\}$, se muestra fácilmente que $\left\{h_{1}^{*}, \ldots, h_{p}^{*}\right\}$ es una base de $G^{\circ}$ y que $\left\{g_{1}^{*}, \ldots, g_{q}^{*}\right\}$ es una base de $H^{\circ}$.

2) Más generalmente, $L=\oplus H_{s}$ y $L^{*}=\oplus H_{s}^{*}$ son dos descomposiciones duales de $L$ y $L^{*}$ si y sólo si $H_{s}^{*}=\left(\bigoplus_{r \neq s} H_{r}\right)^{\circ}=\bigcap_{r \neq s} H_{r}^{\circ}$.

Proposición 3 Las afirmaciones siguientes son equivalentes: 
a) $T$ es no degenerada sobre $H y \operatorname{rang} T=\operatorname{dim} H$.

b) $T_{/ H}$ es inyectiva $y \operatorname{rang} T=\operatorname{dim} H$.

c) $L=H \oplus \operatorname{ker} T$.

d) $L^{*}=\operatorname{Im} T \oplus H^{\circ}$.

Demostración: La equivalencia entre (a) y (b) proviene inmediatamente de la propiedad 1 .

(b) $\Leftrightarrow(\mathrm{c}):$ Como $\operatorname{dim} L=\operatorname{rang} T+\operatorname{dim}(\operatorname{ker} T),(\mathrm{b})$ es equivalente a: $H \cap \operatorname{ker} T=\{0\} \mathrm{y}$ $\operatorname{dim} L=\operatorname{dim} H+\operatorname{dim}(\operatorname{ker} T)$, es decir equivalente a (c).

(c) $\Leftrightarrow\left(\right.$ d): Identificando $L^{* *}$ y $L$, puesto que la forma bilineal $T$ es simétrica, se tiene: $(\operatorname{ker} T)^{\circ}=\operatorname{Im}^{t} T=\operatorname{Im} T$. La equivalence se establece aplicando la propiedad 2.

Se puede notar que las afirmaciones de la proposición 3 también son equivalentes a la afirmación: $T_{/ H}$ es inyectiva y $\operatorname{rang} T_{/ H}=\operatorname{rang} T$.

Corolario 1 Si la forma bilineal simétrica $T$ sobre $L$ es no degenerada sobre un subespacio $H \subset L$, y si $\operatorname{rang} T=\operatorname{dim} H$ entonces:

a) $L=H \oplus \operatorname{ker} T$ y $L^{*}=\operatorname{Im} T \oplus H^{\circ}$ son dos descomposiciones duales de $L$ y $L^{*}$, con $H^{*}=\operatorname{Im} T y(\operatorname{ker} T)^{*}=H^{\circ}$.

b) Se tiene además $\operatorname{ker} T=H^{\perp} y \operatorname{Im} T=\{T(x) / x \in H\}$.

\section{DEMOSTRACIÓN:}

a) La afirmación (a) es una consecuencia directa de la proposición 3 y de la definición de las descomposiciones duales.

b) Se tiene $\operatorname{ker} T \subset H^{\perp}$. Ahora bien, como por hipótesis $T$ es no degenerada sobre $H$, se tiene: $\operatorname{dim} L=\operatorname{dim} H+\operatorname{dim} H^{\perp}=\operatorname{rang} T+\operatorname{dim} H^{\perp}$ puesto que $\operatorname{rang} T=\operatorname{dim} H$, de donde $\operatorname{dim} H^{\perp}=\operatorname{dim}(\operatorname{ker} T)$ y luego $\operatorname{ker} T=H^{\perp}$.

Como $T_{/ H}$ es inyectiva y $\operatorname{rang} T=\operatorname{dim} H$, se tiene: $\operatorname{Im} T=\operatorname{Im} T_{/ H}=\{T(x) / x \in$ $H\}$.

\subsection{Aplicación al Análisis de Datos}

En Análisis de Datos, se dispone de una tabla de datos que contiene la medida de $p$ variables observadas sobre $n$ individuos o unidades estadísticas. A esta tabla le corresponde una matriz con $n$ filas y $p$ columnas, denotada $X$. Al $i$-ésimo individuo (resp. $j$-'esima variable) se le asocia la fila $i$ (resp. la columna $j$ ) de $X$, denotada $x_{i}$ (resp. $x^{j}$ ). El elemento $(i, j)$ de $X$, denotado $x_{i}^{j}$, es el valor de $x^{j}$ observado sobre el individuo $x_{i}$. Así, $x_{i}$ pertenece a un espacio vectorial real de $p$ dimensiones denotado $E$ y llamado espacio de individuos. Igualmente, $x^{j}$ pertenece a un espacio vectorial real de $n$ dimensiones $F$ llamado espacio de variables. 
La matriz $X$ está asociada canónicamente a una aplicación lineal $X: E^{*} \longrightarrow F$ tal que $X\left(e_{j}^{*}\right)=x^{j}$, donde $E^{*}$ es el dual de $E$ y $\left\{e_{j}^{*} / j=1, \ldots, p\right\}$ es la base dual de la base canónica $\left\{e_{j} / j=1, \ldots, p\right\}$ de $E$. La transpuesta de $X$, denotada ${ }^{t} X$, es una aplicación de $F^{*}$ en $E=E^{* *}$ tal que ${ }^{t} X\left(f_{i}^{*}\right)=x_{i}$, donde $F^{*}$ es el dual de $F$ y $\left\{f_{i}^{*}\right\}$ es la base dual de la base canónica $\left\{f_{i}\right\}$ de $F$.

$M$ es una forma bilineal simétrica, no degenerada sobre $\operatorname{Im}{ }^{t} X$, que supondremos positiva y será utilizada posteriormente para definir una distancia entre individuos.

Como $\left(\operatorname{Im}{ }^{t} X\right)^{\circ}=\operatorname{ker} X$, aplicando el corolario 1 se deduce:

Proposición 4 Si la forma bilineal simétrica $M$, no degenerada sobre $\operatorname{Im}{ }^{t} X$, es tal que rang $M=\operatorname{rang} X$, entonces

$$
E=\operatorname{Im}{ }^{t} X \oplus \operatorname{ker} M y E^{*}=\operatorname{Im} M \oplus \operatorname{ker} X
$$

son dos descomposiciones duales de $E$ y $E^{*}$ tales que $\left(\operatorname{Im}{ }^{t} X\right)^{*}=\operatorname{Im} M=\left\{M(x) / x \in \operatorname{Im}^{t} X\right\} y(\operatorname{ker} M)^{*}=\operatorname{ker} X$.

\subsection{La forma bilineal de covarianzas $V$}

Denotamos con la misma letra $D$ una forma bilineal simétrica sobre $F$ y la aplicación lineal de $F$ en $F^{*}$ asociada.

Denotamos $V$ una aplicación de $E^{*} \times E^{*}$ en $\mathbb{R}$ definida por

$$
\forall\left(x^{*}, y^{*}\right) \in E^{*} \times E^{*} \quad V\left(x^{*}, y^{*}\right)=D\left(X x^{*}, X y^{*}\right) .
$$

Es fácil verificar que $V$ es una forma bilineal simétrica sobre $E^{*}$ y que ${ }^{t} X D X$ es una expresión de la aplicación lineal de $E^{*}$ en $E^{* *}=E$, denotada $V$, asociada. Es más, $V$ es positiva si $D$ es positiva.

Se sabe que si la matriz asociada a $D$ en la base canónica de $F$ es la matriz diagonal de pesos de los individuos y si las variables son centradas con respecto a $D$, entonces la matriz asociada a $V$ en la base canónica de $E^{*}$ es la matriz de covarianzas de las variables. $V$ es llamada la forma bilineal de covarianzas.

Recordamos a manera de ilustración el esquema de dualidad, introducido en [1], en la figura 1.

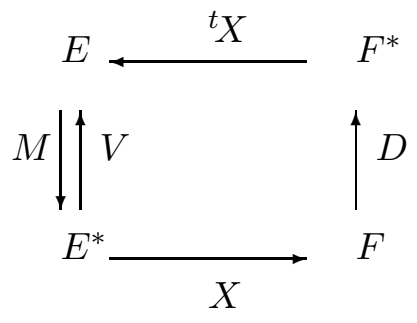

Figura 1: El esquema de dualidad. 
Proposición 5 a) Si $D$ es no degenerada sobre $\operatorname{Im} X$, entonces $\operatorname{ker} V=\operatorname{ker} X$.

b) $\operatorname{ker} V=\operatorname{ker} X$ es equivalente $a \operatorname{Im} V=\operatorname{Im}^{t} X$.

c) Si $\operatorname{ker} V=\operatorname{ker} X$ entonces la restricción de $D a \operatorname{Im} X$, denotada $D_{/ \operatorname{Im} X}$, es inyectiva.

d) Si $D$ es positiva o si $\operatorname{rang} D=\operatorname{rang} X$, entonces una condición necesaria y suficiente para que $D$ sea no degenerada sobre $\operatorname{Im} X$ es que $\operatorname{ker} V=\operatorname{ker} X$.

\section{Demostración:}

a) Se tiene $\operatorname{ker} V \supset \operatorname{ker} X$. Mostremos que $\operatorname{ker} V \subset \operatorname{ker} X:$ Sea $x^{*} \in \operatorname{ker} V, \forall y^{*} \in E^{*}$ se tiene: $D\left(X x^{*}, X y^{*}\right)=\left\langle{ }^{t} X D X x^{*}, y^{*}\right\rangle=\left\langle V x^{*}, y^{*}\right\rangle=0$.

Como por hipótesis $D$ es no degenerada sobre $\operatorname{Im} X$, se deduce que $X\left(x^{*}\right)=0$, es decir $x^{*} \in \operatorname{ker} X$ y entonces $\operatorname{ker} V \subset \operatorname{ker} X$.

b) Se deduce la equivalencia propuesta de:

- $\operatorname{Im} V \subset \operatorname{Im}^{t} X$ y $\operatorname{ker} V \supset \operatorname{ker} X, \mathrm{y}$

- $\operatorname{dim} E^{*}=\operatorname{rang} V+\operatorname{dim}(\operatorname{ker} V)=\operatorname{rang}{ }^{t} X+\operatorname{dim}(\operatorname{ker} X)$.

c) Sea $x \in \operatorname{ker} D / \operatorname{Im} X=\operatorname{Im} X \cap \operatorname{ker} D . \exists y^{*} \in E^{*}$ tal que $x=X\left(y^{*}\right)$. Se tiene entonces $V\left(y^{*}\right)={ }^{t} X D X\left(y^{*}\right)={ }^{t} X D(x)=0$, de donde $y^{*} \in \operatorname{ker} V=\operatorname{ker} X$. Por consiguiente, $x=X\left(y^{*}\right)=0$ y $\operatorname{ker} D / \operatorname{Im} X=\{0\}$.

d) La condición necesaria ha sido establecida en (a), mostremos la condición suficiente. Si se supone que $\operatorname{ker} V=\operatorname{ker} X$ entonces $D_{/ \operatorname{Im} X}$ es inyectiva. Como $D$ es positiva o rang $D=\operatorname{rang} X=\operatorname{dim}(\operatorname{Im} X)$, de la proposición 1 se deduce que $D$ es no degenerada sobre $\operatorname{Im} X$.

Proposición 6 Si $D$ es no degenerada sobre $\operatorname{Im} X$, entonces $V$ es no degenerada sobre todo subespacio suplementario de $\operatorname{ker} X$.

Demostración: Sea $S$ un subespacio suplementario de $\operatorname{ker} X$ en $E^{*}$. Se quiere probar que $V$ es no degenerada sobre $S$, es decir que $\left\{x^{*} \in S / \forall y^{*} \in S, V\left(x^{*}, y^{*}\right)\right\}=0$.

Sea $x^{*} \in S$ tal que $\forall y^{*} \in S \quad V\left(x^{*}, y^{*}\right)=0$. Como $D$ es no degenerada sobre $\operatorname{Im} X$ se tiene $\operatorname{Im} V=\operatorname{Im}^{t} X$, de donde se deduce que $\forall z^{*} \in F^{*}, \exists y^{*} \in S$ tal que ${ }^{t} X\left(z^{*}\right)=V\left(y^{*}\right)$. Se tiene entonces $\forall z^{*} \in F^{*}$ :

$$
\left\langle X x^{*}, z^{*}\right\rangle=\left\langle x^{*}, X z^{*}\right\rangle=\left\langle x^{*}, V y^{*}\right\rangle=V\left(x^{*}, y^{*}\right)=0,
$$

lo que implica que $X\left(x^{*}\right)=0$ o sea $x^{*} \in \operatorname{ker} X$. Como $S \cap \operatorname{ker} X=\{0\}$, se tiene $x^{*}=0$ y $V$ es entonces no degenerada sobre $S$.

En lo que sigue, diremos que $T$ es un semiproducto escalar no degenerado sobre $H$ si $T$ es una forma bilineal sobre $L$, simétrica, positiva y no degenerada sobre $H$. Evidentemente, se trata de un abuso de lenguaje, pero que tiene el fin de simplificar los términos usados. 
Corolario 2 Si $M$ y D son semiproductos escalares no degenerados respectivamente sobre los subespacios $\operatorname{Im}{ }^{t} X y \operatorname{Im} X$, $y$ si rang $M=\operatorname{rang} V$, entonces

a) $V$ es un semiproducto escalar no degenerado sobre $\operatorname{Im} M$.

b) $E=\operatorname{Im} V \oplus \operatorname{ker} M y E^{*}=\operatorname{Im} M \oplus \operatorname{ker} V$ son dos descomposiciones duales de $E$ y $E^{*}$, donde $(\operatorname{Im} V)^{*}=\operatorname{Im} M y(\operatorname{ker} M)^{*}=\operatorname{ker} V$.

\section{Demostración:}

a) Hemos visto que por definición $V$ es bilineal, simétrica y positiva. Mostremos que $V$ es no degenerada sobre $\operatorname{Im} M$. Como por hipótesis $D$ es no degenerada sobre $\operatorname{Im} X$ se tiene (c.f. proposición 5) ker $V=\operatorname{ker} X$ y luego $\operatorname{rang} M=\operatorname{rang} V=\operatorname{rang} X$. Por la proposición 4, Im $M$ es un suplementario de ker $X$ en $E^{*}$, y entonces $V$ es no degenerada sobre $\operatorname{Im} M$, según la proposición anterior.

b) Por la proposición 5 se tiene $\operatorname{ker} V=\operatorname{ker} X$ y $\operatorname{Im} V=\operatorname{Im}{ }^{t} X$, el resultado se establece entonces aplicando la proposición 4.

\subsection{Inversas generalizadas de $V$ y del semiproducto escalar $M$ del espacio de individuos}

SZe supone que $M$ (resp. $D$ ) es un semiproducto escalar no degenerado sobre $\operatorname{Im}{ }^{t} X$ (resp. $\operatorname{Im} X)$ y que rang $M=\operatorname{rang} X$.

Recordamos que una inversa generalizada algebraica, denotada $B^{-}$, de una aplicación lineal $B: L_{1} \longrightarrow L_{2}$, con $L_{1}$ y $L_{2}$ espacios vectoriales, es una aplicación lineal de $L_{2}$ en $L_{1}$ tal que $B^{-} B B^{-}=B^{-}$y $B B^{-} B=B$.

$B^{-}$está caracterizada por $\operatorname{Im} B^{-}$y $\operatorname{ker} B^{-}$, donde estos subespacios son respectivamente los suplementarios de $\operatorname{ker} B$ en $L_{1}$ y $\operatorname{Im} B$ en $L_{2}$.

Por el corolario 2, $E=\operatorname{Im} V \oplus \operatorname{ker} M$ y $E^{*}=\operatorname{Im} M \oplus \operatorname{ker} V$ son dos descomposiciones duales de $E$ y $E^{*}$. Una inversa generalizada algebraica $M^{-}$de $M$ puede entonces ser caracterizada por $\operatorname{Im} M^{-}=\operatorname{Im} V$ y $\operatorname{ker} M^{-}=\operatorname{ker} V$. Notando que la restricción de $M$

a $\operatorname{Im} V$, denotada $M_{/ \operatorname{Im} V}$, es una biyección de $\operatorname{Im} V$ en $\operatorname{Im} M$, se muestra [6] fácilmente (idempotencia de $M M^{-}$o de $M^{-} M$ ) la propiedad siguiente:

Proposición 7 La inversa generalizada algebraica de $M$, especificada por $\operatorname{Im} M^{-}=\operatorname{Im} V$ $y \operatorname{ker} M^{-}=\operatorname{ker} V$, es tal que

$$
\forall x^{*} \in \operatorname{Im} M \quad M^{-}\left(x^{*}\right)=M_{/ \operatorname{Im} V}^{-1}\left(x^{*}\right)
$$

Entonces $E=\operatorname{Im} M^{-} \oplus \operatorname{ker} M$ y $E^{*}=\operatorname{Im} M \oplus \operatorname{ker} M^{-}$son dos descomposiciones duales de $E$ y $E^{*}$ tales que $\left(\operatorname{Im} M^{-}\right)^{*}=\operatorname{Im} M$ y $(\operatorname{ker} M)^{*}=\operatorname{ker} M^{-}$.

Notemos que:

$$
\operatorname{Im} M^{-}=\left\{M^{-}\left(x^{*}\right) / x^{*} \in \operatorname{Im} M\right\}=\operatorname{Im} V=\left\{V\left(x^{*}\right) / x^{*} \in \operatorname{Im} M\right\} .
$$




\section{Proposición 8}

La forma bilineal, denotada $\mathrm{M}^{-}$, definida por

$$
\forall\left(x^{*}, y^{*}\right) \in E^{*} \times E^{*} \quad M^{-}\left(x^{*}, y^{*}\right)=\left\langle x^{*}, M^{-} y^{*}\right\rangle
$$

es un semiproducto escalar no degenerado sobre $\operatorname{Im} M$.

Demostración: $\forall\left(x^{*}, y^{*}\right) \in E^{*} \times E^{*}, x^{*}=x_{1}^{*}+x_{2}^{*}$ y $y^{*}=y_{1}^{*}+y_{2}^{*}$, donde $x_{1}^{*}, y_{1}^{*} \in \operatorname{Im} M$ y $x_{2}^{*}, y_{2}^{*} \in \operatorname{ker} M^{-}$, se tiene:

$$
\begin{aligned}
M^{-}\left(x^{*}, y^{*}\right) & =\left\langle x^{*}, M^{-} y^{*}\right\rangle=\left\langle x_{1}^{*}+x_{2}^{*}, M^{-} y_{1}^{*}\right\rangle \\
& =\left\langle M M^{-} x_{1}^{*}, M^{-} y_{1}^{*}\right\rangle+\left\langle x_{2}^{*}, M^{-} y_{1}^{*}\right\rangle
\end{aligned}
$$

Como $x_{2}^{*} \in \operatorname{ker} M^{-}=\operatorname{ker} V=(\operatorname{Im} V)^{\circ}$ y $\operatorname{Im} M^{-}=\operatorname{Im} V$, se tiene $\left\langle x_{2}^{*}, M^{-} y_{1}^{*}\right\rangle=0 \mathrm{y}$ entonces:

$$
M^{-}\left(x^{*}, y^{*}\right)=M\left(M^{-} x_{1}^{*}, M^{-} y_{1}^{*}\right) .
$$

Como $M$ es simétrica y positiva, también lo es $M^{-}$.

Siendo $M^{-}$positiva y la restricción de $M^{-}$a Im $M$ inyectiva (c.f. proposición 7), por la proposición 1 se obtiene que $M^{-}$es no degenerada sobre $\operatorname{Im} M$.

Así mismo, la inversa generalizada algebraica $V^{-}$de $V$, caracterizada por $\operatorname{ker} V^{-}=\operatorname{ker} M$ y $\operatorname{Im} V^{-}=\operatorname{Im} M$, es tal que

$$
\forall x \in \operatorname{Im} V \quad V^{-}(x)=V_{/ \operatorname{Im} M}^{-1}(x) .
$$

$E=\operatorname{Im} V \oplus \operatorname{ker} V^{-}$y $E^{*}=\operatorname{Im} V^{-} \oplus \operatorname{ker} V$ son entonces dos descomposiciones duales de $E$ y $E^{*}\left(\right.$ c.f. corolario 2) tales que $(\operatorname{Im} V)^{*}=\operatorname{Im} V^{-}$y $\left(\operatorname{ker} V^{-}\right)^{*}=\operatorname{ker} V$.

Es más, se tiene:

1) $\operatorname{Im} V^{-}=\left\{V^{-}(x) / x \in \operatorname{Im} V\right\}=\operatorname{Im} M=\{M(x) / x \in \operatorname{Im} V\}$.

2) La forma bilineal, denotada $V^{-}$, definida por:

$$
\forall(x, y) \in E \times E \quad V^{-}(x, y)=\left\langle x, V^{-} y\right\rangle
$$

es un semiproducto escalar no degenerado sobre $\operatorname{Im} V$.

Definición 1 Cuando $D$ es el producto escalar de pesos se dice que $V^{-}$es un semiproducto escalar de Mahalanobis.

\section{Operadores útiles en Análisis de Datos}

En las dos partes de esta sección se supone que $M$ y $D$ son semiproductos escalares no degenerados respectivamente sobre $\operatorname{Im}{ }^{t} X$ y $\operatorname{Im} X$, y que $\operatorname{rang} M=\operatorname{rang} X$. Se tiene entonces también rang $M=\operatorname{rang} V$ (c.f. proposición 5). 


\section{$3.1 \quad$ El operador $V M$}

En esta subsección estudiamos algunas propiedades del operador $V M$ sobre E. Estas propiedades serán importantes más adelante donde se recordarán las definiciones de la inercia y de los ejes principales de dispersión de una nube de puntos. Así mismo, este operador será utilizado en expresiones de los productos escalares relacionales.

Recordemos algunas definiciones y propiedades generales en el caso en que $L$ es un espacio vectorial de dimensión finita y $T$ un semiproducto escalar no degenerado sobre el subespacio $H \subset L$. Diremos que el operador $A$ sobre $L$ es

- $T$-simétrico si $\forall(x, y) \in L \times L \quad T(A x, y)=T(x, A y)$

- $T$-positivo si $\forall(x, x) \in L \times L \quad T(A x, x) \geq 0$.

Observación: En presencia de semiproductos escalares, no se garantiza la existencia y la unicidad de la adjunta de un operador $A$. Denotando $T$ a la aplicación lineal de $L$ en $L^{*}$ asociada a $T$, una condición suficiente de existencia es que $\operatorname{Im}\left({ }^{t} A T\right) \subset \operatorname{Im} T$. En efecto, en este caso si $T^{-}$es una inversa generalizada interna de $T$, entonces:

$$
\forall(x, y) \in L \times L \text { se tiene } T(A x, y)=\left\langle x,{ }^{t} A T y\right\rangle=\left\langle x, T T^{-t} A T y\right\rangle=T\left(x, T^{-t} A T y\right)
$$

pues $T T^{-}$es un proyector sobre $\operatorname{Im} T$, lo que prueba la existencia de la adjunta $A^{*}=T^{-t} A T$ de $A$.

Notemos que $V M$ satisface esta condición, pues $\operatorname{Im}^{t}(V M) M=\operatorname{Im} M V M \subset \operatorname{Im} M$.

Sea $P: L \longrightarrow \operatorname{Im} P \subset H \subset L$ una aplicación tal que $\forall x \in \operatorname{Im} P \quad P(x)=x$ y ker $P=(\operatorname{Im} P)^{\perp}$. Notemos que $L=\operatorname{Im} P \oplus(\operatorname{Im} P)^{\perp}$ puesto que, $T_{/ H}$ y por tanto $T_{/ \operatorname{Im} P}$ siendo inyectivas (c.f. proposición 1(a)) y $T$ positiva, $T$ es no degenerada sobre $\operatorname{Im} P$ (c.f. proposición 1(c)). Por lo tanto es natural llamar $P$ el operador de proyección ortogonal sobre $\operatorname{Im} P$ respecto a $T$.

Proposición 9 Como $T$ es un semiproducto escalar no degenerado sobre $H$, las siguientes afirmaciones son equivalentes:

a) $P$ es un operador de proyección ortogonal sobre $\operatorname{Im} P$ respecto a $T$

b) Im $P \subset H, P$ es idempotente y T-simétrico.

\section{Demostración:}

a) Rightarrow b): Supongamos que $P$ es un operador de proyección ortogonal. Se tiene $\operatorname{Im} P \subset H$ por definición de $P . P$ es idempotent pues $\forall x \in L, x=x_{1}+x_{2}$ donde $x_{1} \in \operatorname{Im} P$ y $x_{2} \in \operatorname{ker} P$, se tiene: $P^{2}(x)=P^{2}\left(x_{1}\right)=P\left(x_{1}\right)=P(x)$.

Mostremos que $P$ es $T$-simétrico:

$\forall(x, y) \in L \times L, x=x_{1}+x_{2}$ y $y=y_{1}+y_{2}$ donde $x_{1}, y_{1} \in \operatorname{Im} P$ y $x_{2}, y_{2} \in \operatorname{ker} P$, se tiene:

$$
\begin{aligned}
T(P x, y) & =T\left[P\left(x_{1}+x_{2}\right), y_{1}+y_{2}\right] \\
& =T\left(x_{1}, y_{1}+y_{2}\right) \\
& =T\left(x_{1}, y_{1}\right) \text { pues } \operatorname{ker} P=(\operatorname{Im} P)^{\perp}
\end{aligned}
$$

e igualmente $T(x, P y)=T\left(x_{1}, y_{1}\right)$.

Se tiene entonces $T(P x, y)=T(x, P y)$. 
b) Rightarrow a): $\forall x_{1} \in \operatorname{Im} P, \exists y \in L$ tal que $P(y)=x_{1}$. Como por hipótesis $P$ es idempotente, se tiene $P\left(x_{1}\right)=P P(y)=P(y)=x_{1}$. Es más, $\forall x_{2} \in \operatorname{ker} P, \forall z \in \operatorname{Im} P$ se tiene $T\left(x_{2}, z\right)=T\left(x_{2}, P z\right)=T\left(P x_{2}, z\right)=0$ pues $P$ es $T$-simétrico, entonces ker $P=(\operatorname{Im} P)^{\perp}$.

Como $\operatorname{Im} P \subset H$ por hipótesis, $P$ es, por definición, un operador de proyección ortogonal.

Observación: Se puede mostrar que

$\forall x \in L \quad T(x-P x, x-P x)=\min _{y \in \operatorname{Im} P} T(x-y, x-y)$.

Observación: En el caso en que $M$ es un semiproducto escalar no degenerado sobre Im ${ }^{t} X$ tal que rang $M=\operatorname{rang} X, M^{-} M$ es un operador de proyección ortogonal sobre $\operatorname{Im}{ }^{t} X$ respecto a $M$ y $M M^{-}$lo es sobre $\operatorname{Im} M$ respecto a $M^{-}$.

Proposición 10 Como $M$ y $D$ son semiproductos escalares no degenerados respectivamente sobre $\operatorname{Im}{ }^{t} X y \operatorname{Im} X, y$ como $\operatorname{rang} M=\operatorname{rang} X$, se tiene:

a) La restricción de $V M a \operatorname{Im}{ }^{t} X$, denotada $V M_{/ \operatorname{Im}}{ }^{t} X$, es inyectiva.

b) $\operatorname{Im} V M=\operatorname{Im}{ }^{t} X$.

c) $\operatorname{ker} V M=\operatorname{ker} M$.

d) $V M$ es $M$-simétrico y $M$-positivo.

DEMOSTRACIÓN:

a) Como por hipótesis $M$ es no degenerada sobre $\operatorname{Im}{ }^{t} X$ y $V$ es no degenerada sobre

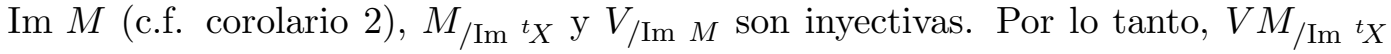
es inyectiva.

b) Se tiene $\operatorname{Im} V M \subset \operatorname{Im} V=\operatorname{Im}^{t} X$ y $\operatorname{rang}\left(V M_{/ \operatorname{Im}}{ }^{t} X\right)=\operatorname{rang}{ }^{t} X$. Puesto que rang $V M \geq \operatorname{rang}\left(V M_{/ \operatorname{Im}}{ }^{t} X\right)$ se tiene rang $V M \geq \operatorname{rang}{ }^{t} X$ y luego $\operatorname{Im} V M=\operatorname{Im}{ }^{t} X$.

c) Se tiene $\operatorname{ker} V M \supset \operatorname{ker} M$. Además $\operatorname{rang} V M=\operatorname{rang} V=\operatorname{rang} M$, entonces $\operatorname{dim}(\operatorname{ker} V M)=\operatorname{dim}(\operatorname{ker} M)$ y $\operatorname{ker} V M=\operatorname{ker} M$.

d) Como $V$ es un semiproducto escalar se tiene ${ }^{t} V=V$ al identificar $E^{* *}$ y $E$, de donde $\forall(x, y) \in E \times E$ se tiene:

$$
M(V M x, y)=\langle V M x, M y\rangle=\langle M x, V M y\rangle=M(x, V M y)=V(M x, M y) .
$$

Por lo tanto $V M$ es $M$-simétrico y $M$-positivo.

Según la igualdad $E=\operatorname{Im}{ }^{t} X \oplus \operatorname{ker} M$ y los incisos (b) y (c) de la proposición anterior, podemos escribir $E=\operatorname{Im} V M \oplus \operatorname{ker} V M$.

Denotamos $\widetilde{\operatorname{Im}^{t} X}$ el espacio vectorial canónicamente isomorfo a $\operatorname{Im}{ }^{t} X$. Sean $\operatorname{pr}_{t} X$ : $\operatorname{Im}^{t} X \subseteq E \longrightarrow \widetilde{\operatorname{Im}^{t} X}$ la proyección canónica de $\operatorname{Im}^{t} X$ sobre $\widetilde{\operatorname{Im}^{t} X} \mathrm{y}$ $i n_{t} X: \widetilde{\operatorname{Im}^{t} X} \longrightarrow \operatorname{Im}^{t} X \subset E$ la inyección canónica de $\widetilde{\operatorname{Im}^{t} X}$ en $\operatorname{Im}{ }^{t} X$. 
Definimos la forma bilineal $\widetilde{M}$ sobre $\widetilde{\operatorname{Im}^{t} X}$ por:

$$
\forall(\tilde{x}, \tilde{y}) \in \widetilde{\operatorname{Im}^{t} X} \times \widetilde{\operatorname{Im}^{t} X} \widetilde{M}(\tilde{x}, \tilde{y})=M(x, y)
$$

donde $x=i n_{t_{X}}(\tilde{x})$ y $y=i n_{t_{X}}(\tilde{y})$.

Proposición $11 \widetilde{M}$ es un producto escalar sobre $\widetilde{\operatorname{Im}^{t} X}$ y una expresión de la aplicación lineal asociada, también denotada $\widetilde{M}$, es $\widetilde{M}={ }^{t} i n_{t_{X}} M i n_{t} X$.

Demostración: La bilinealidad de $\widetilde{M}$ se deduce de la de $M$ y de la linealidad de $i n_{t} X$. La simetría y la positividad se deduicen de las de $M$. Mostremos que $\widetilde{M}$ es no degenerada sobre el espacio $\widetilde{\operatorname{Im}^{t} X}$.

Sea $\tilde{x} \in \widetilde{\operatorname{Im}^{t} X}$ tal que $\forall \tilde{y} \in \widetilde{\operatorname{Im}^{t} X} M(x, y)=\widetilde{M}(\tilde{x}, \tilde{y})=0$, donde $x=i n_{t} X(\tilde{x})$ y $y=i n_{t}(\tilde{y})$. Como $M$ es no degenerada sobre $\operatorname{Im}^{t} X$, se tiene $x=0$ y por consiguiente $\tilde{x}=p r^{t_{X}}(x)=0$. Por lo tanto $\widetilde{M}$ es no degenerado.

Mostremos ahora que $\widetilde{M}={ }^{t} i n_{t}{ }_{X} M^{i} n_{t}$ es una expresión de la aplicación lineal asociada a $\widetilde{M}$.

$\forall(\tilde{x}, \tilde{y}) \in \widetilde{\operatorname{Im}^{t} X} \times \widetilde{\operatorname{Im}^{t} X}, x=i n_{t} X(\tilde{x})$ y $y=i n_{t} X(\tilde{y})$, se tiene:

$$
\begin{aligned}
\widetilde{M}(\tilde{x}, \tilde{y})=M(x, y) & =M\left(i n_{t_{X}} \tilde{x}, i n_{t_{X}} \tilde{y}\right) \\
& =\left\langle i n_{t_{X}} \tilde{x}, \operatorname{Min}_{t_{X}} \tilde{y}\right\rangle \\
& =\left\langle\tilde{x}^{t}{ }^{t} n_{t_{X}} \operatorname{Min}_{t_{X}} \tilde{y}\right\rangle
\end{aligned}
$$

Proposición 12 El operador $\widetilde{V M}$ sobre $\widetilde{\operatorname{Im}^{t} X}$ definido por $\widetilde{V M}=p r_{t_{X}} V M^{2} i_{t_{X}}$ es $\widetilde{M}$-simétrico y $\widetilde{M}$-positivo.

Demostración: $\forall(\tilde{x}, \tilde{y}) \in \widetilde{\operatorname{Im}^{t} X} \times \widetilde{\operatorname{Im}^{t} X}, x=i n_{t}(\tilde{x})$ y $y=i n_{t} X(\tilde{y})$, se tiene:

$$
\begin{aligned}
\widetilde{M}(\widetilde{V M} \tilde{x}, \tilde{y}) & =\widetilde{M}\left(p_{t_{X}} V \operatorname{Min}_{t_{X}} \tilde{x}, \tilde{y}\right) \\
& =M\left(i_{t_{X}} \operatorname{pr}_{t_{X}} V M x, y\right) \\
& =M(V M x, y) .
\end{aligned}
$$

Como $V M$ es $M$-simétrico y $M$-positivo, entonces $\widetilde{V M}$ es $\widetilde{M}$-simétrico y $\widetilde{M}$-positivo.

Proposición 13 El operador $V M$ tiene rang $X$ valores propios positivos no nulos $y$ Im $V M$ es suma directa de los subespacios propios $M$-ortogonales asociados a estos valores propios. El subespacio propio associado al valor propio nulo, si existe, es $\operatorname{ker} V M$.

Demostración: Como $\widetilde{M}$ es un producto escalar sobre $\widetilde{\operatorname{Im}^{t} X}$ y $\widetilde{V M}$ un operador $\widetilde{M}$ simétrico y $\widetilde{M}$-positivo, $\widetilde{V M}$ tiene rang $\widetilde{V M}=\operatorname{rang} V M=\operatorname{rang} X$ valores propios estrictamente positivos.

Sea $\left\{\lambda_{j} / j=1, \ldots\right.$, rang $\left.X\right\}$ el conjunto de valores propios no nulos de $\widetilde{V M}$ y 
$\left\{\tilde{u}_{j} / j=1, \ldots, \operatorname{rang} X\right\}$ el conjunto de vectores propios asociados. Para todo $j \in\{1, \ldots$, rang $X\}$ se tiene

$$
V \operatorname{Min}_{t_{X}} \tilde{u}_{j}=i n_{t_{X}} \operatorname{pr}_{t_{X}} V M i n_{t_{X}} \tilde{u}_{j}=i n_{t} \widehat{V M} \tilde{u}_{j}=\lambda_{j} i n_{t_{X}} \tilde{u}_{j} .
$$

Si ponemos $u_{j}=i n_{t}\left(\tilde{u}_{j}\right)$ se obtiene $V M\left(u_{j}\right)=\lambda_{j} u_{j}$. Los $u_{j}$ sont por lo tanto vectores propios de $V M$ asociados a los valores propios positivos $\lambda_{j}$. Como los $\tilde{u}_{j}$ asociados a valores propios diferentes son $\widetilde{M}$-ortogonales, los $u_{j}$ correspondientes son $M$-ortogonales pues $M\left(u_{j}, u_{k}\right)=\widetilde{M}\left(\tilde{u}_{j}, \tilde{u}_{k}\right)$.

Debido a la propiedad 10 , los $u_{j}$ pertenecen a $\operatorname{Im} V M\left(=\operatorname{Im}{ }^{t} X\right)$ y por tanto $\operatorname{Im} V M$ es suma directa de los subespacios propios $M$-ortogonales asociados a los valores propios no nulos de $V M$.

Comme $E=\operatorname{Im} V M \oplus \operatorname{ker} V M, \operatorname{ker} V M(=\operatorname{ker} M)$ es el subespacio propio asociado al valor propio nulo, si éste existe.

Por la proposición 12 , se tiene la descomposición espectral $\widetilde{V M}=\sum_{j=1}^{\operatorname{rang} X} \lambda_{j} \widetilde{P}_{j}$ de $\widetilde{V M}$, donde $\widetilde{P}_{j}$ es el operador de proyección $\widetilde{M}$-ortogonal sobre el subespacio propio asociado al valor propio $\lambda_{j}$.

Proposición 14 Si $\forall x \in \operatorname{Im}^{t} X \quad P_{j}(x)=i n_{t}{ }_{X} \widetilde{P}_{j} \operatorname{pr}_{t}(x)$

$$
\forall x \in\left(\operatorname{Im}^{t} X\right)^{\perp} \quad P_{j}(x)=0
$$

entonces $P_{j}$ es el operador de proyección $M$-ortogonal sobre el subespacio propio de $V M$ asociado a $\lambda_{j}$ y $V M=\sum_{j=1}^{\mathrm{rang} X}{ }_{\lambda_{j}} P_{j}$ es la descomposición espectral de $V M$ respecto a $M$.

Demostración: Por definición de $P_{j}$ se tiene $\operatorname{Im} P_{j} \subset \operatorname{Im}{ }^{t} X$.

$P_{j}$ es idempotente pues $\forall x \in E \quad x=x_{1}+x_{2}$ con $x_{1} \in \operatorname{Im}^{t} X$ y $x_{2} \in\left(\operatorname{Im}{ }^{t} X\right)^{\perp}$, se tiene

$$
\begin{aligned}
P_{j}^{2}(x)=P_{j}^{2}\left(x_{1}\right) & =i n_{t_{X}} \widetilde{P}_{j} p r_{t_{X}} i n_{t_{X}} \widetilde{P}_{j} p r_{t_{X}}\left(x_{1}\right) \\
& =i n_{t_{X}} \widetilde{P}_{j}^{2} p r_{t_{X}}\left(x_{1}\right) \\
& =i n_{t_{X}} \widetilde{P}_{j} p r_{t_{X}}\left(x_{1}\right)=P_{j}(x) .
\end{aligned}
$$

Mostremos que $P_{j}$ es $M$-simétrico: $\forall(x, y) \in E \times E$ tal que $x=x_{1}+x_{2}$ y $y=y_{1}+y_{2}$, donde $x_{1}, y_{1} \in \operatorname{Im}^{t} X$ y $x_{2}, y_{2} \in \operatorname{ker} M=\left(\operatorname{Im}^{t} X\right)^{\perp}$, se tiene $M\left(P_{j} x, y\right)=M\left(P_{j} x_{1}, y_{1}\right)$. Además, por definición de $\widetilde{M}$ y en denotando $\tilde{x}_{1}=p r_{t_{X}}\left(x_{1}\right)$ y $\tilde{y}_{1}=p r_{t_{X}}\left(y_{1}\right)$, se tiene:

$$
\begin{aligned}
M\left(P_{j} x_{1}, y_{1}\right) & =\left\langle M i n_{t_{X}} p r_{t_{X}} P_{j} x_{1}, i n_{t_{X}} p r_{t_{X}} y_{1}\right\rangle \\
& =\widetilde{M}\left(p r_{t_{X}} P_{j} x_{1}, p r_{t_{X}} y_{1}\right) \\
& =\widetilde{M}\left(\widetilde{P}_{j} \tilde{x}_{1}, \tilde{y}_{1}\right) .
\end{aligned}
$$

Así mismo, $M\left(x, P_{j} y\right)=\widetilde{M}\left(\tilde{x}_{1}, \widetilde{P}_{j} \tilde{y}_{1}\right)$ de donde el resultado puesto que $\widetilde{P}_{j}$ es $\widetilde{M}$-simétrico. Ahora bien, se tiene

$$
V M=i n_{t} \widetilde{V M} p r_{t}
$$




$$
\begin{aligned}
& =i n_{t_{X}}\left(\sum_{j=1}^{\operatorname{rang} X} \lambda_{j} \widetilde{P}_{j}\right) p r_{t_{X}} \\
& =\sum_{j=1}^{\operatorname{rang} X} \lambda_{j}\left(i n_{t_{X}} \widetilde{P}_{j} p r_{t_{X}} .\right.
\end{aligned}
$$

Se tiene entonces $V M=\sum_{j=1}^{\mathrm{rang} X} \lambda_{j} P_{j}$.

Observación: Los $P_{j}$ son $M$-positivos: $\forall x \in E$, como los $P_{j}$ son $M$-simétricas e idempotentes, se tiene: $M\left(P_{j} x, x\right)=M\left(P_{j}^{2} x, x\right)=M\left(P_{j} x, P_{j} x\right) \geq 0$.

Proposición 15 Si $M V M$ es la forma bilineal definida por

$$
\forall(x, y) \in E \times E \quad M V M(x, y)=\langle x, M V M y\rangle
$$

entonces $M V M$ es un semiproducto escalar no degenerado sobre $\operatorname{Im}{ }^{t} X$.

Demostración: $M V M$ es simétrica (resp. positiva) pues $M$ es simétrica (resp. positiva) y $V M$ es $M$-simétrica (resp. $M$-positiva). Como $M$ es no degenerada sobre $\operatorname{Im}^{t} X$, la restricción de $M$ a $\operatorname{Im}{ }^{t} X$ es inyectiva (c.f. proposición 1). De la inyectividad de $V M / \operatorname{Im}{ }^{t} X$ y de $\operatorname{Im}(V M)=\operatorname{Im}{ }^{t} X$ (c.f. proposición 10), se deduce que la restricción de la aplicación compuesta $M V M$ a $\operatorname{Im}^{t} X$ es inyectiva.

La forma bilineal $M V M$, siendo positiva, por la proposición 1 es no degenerada sobre $\operatorname{Im}{ }^{t} X$.

Es fácil ver, como para la proposición 7, que la inversa generalizada algebraica $(V M)^{-}$ de $V M$, caracterizada por $\operatorname{ker}(V M)^{-}=\operatorname{ker} V M(=\operatorname{ker} M)$ y $\operatorname{Im}(V M)^{-}=\operatorname{Im} V M$ $\left(=\operatorname{Im}{ }^{t} X\right)$, es tal que: $\forall x \in \operatorname{Im}^{t} X(V M)^{-}(x)=V M_{/ \operatorname{Im}{ }^{t} X}^{-1}(x)$.

Observación: Por abuso de languaje, se puede decir que $(V M)^{-}$es una inversa generalizada de $V M$ ponderada por el semiproducto escalar $M$. En efecto, como $(V M)^{-} V M$ y $V M(V M)^{-}$son proyectores, entonces son operadores idempotentes; además, se tiene $\operatorname{Im}(V M)^{-} V M=\operatorname{Im} V M(V M)^{-}=\operatorname{Im}^{t} X$ y $\operatorname{ker}(V M)^{-} V M=\operatorname{ker} V M(V M)^{-}=\operatorname{ker} M=$ $\left(\operatorname{Im}{ }^{t} X\right)^{\perp}$. Luego, $(V M)^{-} V M$ y $V M(V M)^{-}$son operadores de proyección ortogonal sobre $\operatorname{Im}{ }^{t} X$ respecto a $M$. Esta propiedad es característica de las inverses generalizadas ponderadas por un producto escalar, en el sentido de Chipman [6].

Proposición 16 El operador $(V M)^{-}$es $M$-simétrico y $M$-positivo.

Demostración: Mostremos que $(V M)^{-}$es $M$-simétrico. $\forall(x, y) \in E \times E, x=x_{1}+x_{2}$ y $y=y_{1}+y_{2}$ donde $x_{1}, y_{1} \in \operatorname{Im}^{t} X$ y $x_{2}, y_{2} \in \operatorname{ker} M$, como $V M$ es $M$-simétrico y $V M(V M)^{-}$ es un operador de proyección sobre $\operatorname{Im}^{t} X$ se tiene:

$$
\begin{aligned}
M\left[(V M)^{-} x, y\right] & =M\left[(V M)^{-} x_{1}, y_{1}\right]=M\left[(V M)^{-} x_{1}, V M(V M)^{-} y_{1}\right] \\
& =M\left[V M(V M)^{-} x_{1},(V M)^{-} y_{1}\right]=M\left[x_{1},(V M)^{-} y_{1}\right] \\
& =M\left[x,(V M)^{-} y\right]
\end{aligned}
$$


de donde $(V M)^{-}$es $M$-simétrico.

La $M$-positividad de $(V M)^{-}$es inducida en la tercera igualdad por la $M$-positividad de $V M$.

Podemos notar que, si $u_{j}$ es un vector propio de $V M$ asociado a una valor propio no nulo $\lambda_{j}$, entonces $u_{j}$ también es vector propio de $(V M)^{-}$asociado al valor propio $1 / \lambda_{j}$ : en efecto, puesto que $u_{j} \in \operatorname{Im}^{t} X$ se tiene: $u_{j}=(V M)^{-} V M\left(u_{j}\right)=\lambda_{j}(V M)^{-}\left(u_{j}\right)$. De allí se deduce:

Proposición 17 El operador $(V M)^{-}$tiene rang $X$ valores propios positivos no nulos, inversos de los de $V M$. Los subespacios propios ortogonales asociados son idénticos a los de $V M y \operatorname{ker}(V M)^{-}(=\operatorname{ker} M=\operatorname{ker} V M)$ es el subespacio propio asociado al valor propio nulo, si éste existe.

\section{$3.2 \quad$ El operador $M V$}

Proposición 18 Siendo $M$ y D semiproductos escalares no degenerados, respectivamente sobre $\operatorname{Im}{ }^{t} X y \operatorname{Im} X, y$ como $\operatorname{rang} M=\operatorname{rang} X$, se tiene:

a) La restricción de $M V$ a $\operatorname{Im} M$ es inyectiva.

b) $\operatorname{Im} M V=\left\{M(x) / x \in \operatorname{Im}^{t} X\right\}=\operatorname{Im} M$.

c) $\operatorname{ker} M V=\operatorname{ker} X$.

d) $M V$ es $M^{-}$-simétrico y $M^{-}$-positivo.

Demostración:

a) Como $M$ y $V$ son no degeneradas sobre $\operatorname{Im}{ }^{t} X=\operatorname{Im} V$ y $\operatorname{Im} M$, respecticamente (c.f. corolario 2 pues rang $V=\operatorname{rang} X$ según la proposición 5 ), entonces $M_{/ \operatorname{Im} V} \mathrm{y}$ $V_{/ \operatorname{Im} M}$ son inyectivas. Se deduce luego la inyectividad de la restricción de $M V$ a $\operatorname{Im} M$.

b) En efecto, se tiene $\operatorname{Im} V=\operatorname{Im}{ }^{t} X$ y $\operatorname{Im} M=\left\{M(x) / x \in \operatorname{Im}{ }^{t} X\right\}$ (c.f. proposición 4).

c) Se tiene $\operatorname{ker} M V \supset \operatorname{ker} X$, pues $\operatorname{ker} V=\operatorname{ker} X$ (proposición 5). Además se deduce de (b) que rang $M V=\operatorname{rang} M$ y por hipótesis $\operatorname{rang} M=\operatorname{rang} X$, de donde $\operatorname{dim}(\operatorname{ker} M V)=\operatorname{dim}(\operatorname{ker} X)$ y por tanto $\operatorname{ker} M V=\operatorname{ker} X$.

d) $\forall\left(x^{*}, y^{*}\right) \in E^{*} \times E^{*}$, puesto que $M^{-}$y $V$ son formas bilineales simétricas, $M^{-} M$ un operador de proyección sobre $\operatorname{Im} M^{-}=\operatorname{Im} V=\operatorname{Im}{ }^{t} X$, se tiene:

$$
\begin{aligned}
M^{-}\left(M V x^{*}, y^{*}\right) & =\left\langle M^{-} M V x^{*}, y^{*}\right\rangle=\left\langle V x^{*}, y^{*}\right\rangle \\
& =V\left(x^{*}, y^{*}\right)=\left\langle x^{*}, V y^{*}\right\rangle \\
& =\left\langle x^{*}, M^{-} M V y^{*}\right\rangle=M^{-}\left(x^{*}, M V y^{*}\right) .
\end{aligned}
$$

Por lo tanto, $M V$ es $M^{-}$-simétrica y $M^{-}$-positiva puesto que $V$ es positiva. 
Si $u_{j}$ es un vector propio de $V M$ asociado a $\lambda_{j}>0$, se tiene: $M V\left[M\left(u_{j}\right)\right]=\lambda_{j} M\left(u_{j}\right)$. Puesto que $u_{j} \in \operatorname{Im}^{t} X$ y que $M$ es inyectiva sobre $\operatorname{Im}{ }^{t} X, M\left(u_{j}\right)$ es vector propio de $M V$ asociado a $\lambda_{j}$. Los subespacios propios de $M V$ asociados a los $\lambda_{j}>0$ son entonces las imágenes por $M$ de los subespacios propios de $V M$ y $\operatorname{Im} M$ en es la suma directa. Es más, puesto que $E^{*}=\operatorname{Im} M \oplus \operatorname{ker} X, \operatorname{Im} M V=\operatorname{Im} M, \operatorname{ker} M V=\operatorname{ker} X \mathrm{y}$ $\forall x, y \in \operatorname{Im}^{t} X \quad M(x, y)=M^{-}(M x, M y)$, se deduce:

Proposición 19 El operador $M V$ tiene rang $X$ valores propios positivos no nulos, idénticos a los de $V M$, y los vectores propios asociados son las imágenes por $M$ de los vectores propios de $V M$. Los subespacios propios de $M V$ son $M^{-}$-ortogonales y $\operatorname{Im} M V$ en es la suma directa. El subespacio propio asociado al valor propio nulo, si existe, es ker $M V$.

\section{Aplicación al Análisis de Datos}

\subsection{Inercia, momento de inercia y producto de inercia}

El triplete $(X, M, D)$ caracteriza la nube de puntos individuos $\left\{x_{1}, \ldots, x_{n}\right\}$, denotada $\mathcal{N}$. Como las variables se suponen centradas, el centro de gravedad de la nube $\mathcal{N}$ es el origen de coordenadas de $E$. Puesto que la restricción de $M$ a $\operatorname{Im}{ }^{t} X$ es un producto escalar y como $\forall i \quad x_{i} \in \operatorname{Im}^{t} X$, las expresiones clásicas [1] de la inercia, del momento de inercia y del producto de inercia de $\mathcal{N}$ siguen siendo válidas, pero tomando encuenta algunas precauciones. Por ejemplo, si $H$ es un subespacio de $\operatorname{Im}{ }^{t} X$, no se podrá hablar de la proyección ortogonal sobre $H^{\perp}$ pues $H^{\perp}$ no es necesariamente no isotrópico.

Recordemos las definiciones y expresiones de estos índices:

a) La inercia de la nube $\mathcal{N}$ respecto a su centro de gravedad es:

$$
I[\mathcal{N}]=\sum_{i=1}^{n} p_{i}\left\|x_{i}\right\|_{M}^{2}=\operatorname{traza}(V M)
$$

donde $V$ es la matriz des covarianzas de las variables.

b) Si $H$ es un subespacio vectorial de $\operatorname{Im}{ }^{t} X$ y $P_{H}$ es el operador de proyección ortogonal sobre $H$ respecto a $M$, el momento de inercia de $\mathcal{N}$ respecto a $H$ es (c.f. ver nota en página 44):

$$
I_{H}[\mathcal{N}]=\sum_{i=1}^{n} p_{i}\left\|x_{i}-P_{H}\left(x_{i}\right)\right\|_{M}^{2}
$$

c) Sean $u$ y $v$ dos vectores de $\operatorname{Im}{ }^{t} X, \Delta u$ y $\Delta v$ las rectas que generan, si se denota $(\Delta u)^{\perp}$ $\mathrm{y}(\Delta v)^{\perp}$ los subespacios ortogonales respectivos de $\Delta u$ y $\Delta v$, entonces el producto de inercia de $\mathcal{N}$ respecto a $(\Delta u)^{\perp}$ y $(\Delta v)^{\perp}$ es:

$$
P I\left[\mathcal{N} /(\Delta u)^{\perp} \times(\Delta v)^{\perp}\right]=\sum_{i=1}^{n} p_{i} \frac{M\left(x_{i}, u\right)}{\|u\|_{M}} \frac{M\left(x_{i}, v\right)}{\|v\|_{M}}=\frac{M V M(u, v)}{\|u\|_{M}\|v\|_{M}} .
$$


Obsérvese que, como $P_{\Delta u}$ es el operador de proyección ortogonal sobre $\Delta u$, se tiene:

$$
I\left[P_{\Delta u}(\mathcal{N})\right]=P I\left[\mathcal{N} /(\Delta u)^{\perp} \times(\Delta u)^{\perp}\right]=\frac{M V M(u, u)}{\|u\|_{M}^{2}} .
$$

\subsection{Análisis en Componentes Principales}

El espacio $E$ de individuos está provisto de un semiproducto escalar $M$, que suponemos no degenerado sobre $\operatorname{Im}^{t} X$ y tal que $\operatorname{rang} M=\operatorname{rang} X$. El espacio $F$ de las variables está provisto del semiproducto escalar de pesos $D$, definido por $D\left(f_{j}, f_{k}\right)=p_{j} \delta_{j k}$, donde $p_{j} \geq 0$ y $\sum_{j=1}^{n} p_{j}=1$, y lo suponemos no degenerado sobre $\operatorname{Im} X$. Es más, en lo que sigue supondremos que las variables están centradas.

El hecho de dotar de pesos nulos a algunos individuos, corresponde generalmente a la técnica de definir individuos suplementarios en Análisis de Datos (c.f. por ejemplo [2]).

Proposición 20 a) Una condición necesaria y suficiente para que $D$ sea no degenerado sobre $\operatorname{Im} X$ es que $\operatorname{Im} X \cap \operatorname{ker} D=\{0\}$.

b) Notemos $K$ el subconjunto de $\{1, \ldots, n\}$ tal que $\forall k \in K \quad p_{k}=0$ y $\forall \ell \notin K \quad p_{\ell}>0, y$ $\left\langle f_{k} / k \in K\right\rangle$ el subespacio de $F$ generado por $\left\{f_{k} / k \in K\right\}$. Se tiene $\operatorname{Im} X \cap \operatorname{ker} D=\{0\}$ si y sólo si $\operatorname{Im} X \cap\left\langle f_{k} / k \in K\right\rangle=\{0\}$.

\section{DEMOSTRACIÓN:}

a) Puesto que la forma bilineal simétrica $D$ es positiva, el semiproducto escalar $D$ es no degenerado sobre $\operatorname{Im} X$ si y sólo si la restricción de $D$ a $\operatorname{Im} X$ es inyectiva (c.f. proposición $1(\mathrm{a})$ y (c)), lo que es equivalente a decir que $\operatorname{Im} X \cap \operatorname{ker} D=\{0\}$.

b) Mostremos que $\operatorname{ker} D=\left\langle f_{k} / k \in K\right\rangle: \forall x=\sum_{i=1}^{n} x_{i} f_{i} \in \operatorname{ker} D$ se tiene $\sum_{\ell \notin K} p_{\ell}\left(x_{\ell}\right)^{2}=\sum_{i=1}^{n} p_{i}\left(x_{i}\right)^{2}=D(x, x)=0$, ahora bien $\forall \ell \notin K \quad p_{\ell}>0$, luego $\forall \ell \notin K \quad x_{\ell}=0$ lo que implica $x \in\left\langle f_{k} / k \in K\right\rangle$.

Inversamente, $\forall x=\sum_{k \in K} x_{k} f_{k} \in\left\langle f_{k} / k \in K\right\rangle$ se tiene $D(x, x)=\sum_{k \in K} p_{k}\left(x_{k}\right)^{2}=0$ pues $\forall k \in K \quad p_{k}=0 ; x$ es entonces isotrópico y como $D$ es positiva, $x \in \operatorname{ker} D$.

Se tiene por lo tanto $\operatorname{Im} X \cap \operatorname{ker} D=\{0\}$ si y sólo si $\operatorname{Im} X \cap\left\langle f_{k} / k \in K\right\rangle=\{0\}$.

En presencia de pesos nulos, las condiciones necesarias y suficientes establecidas en la propiedad 20, muestran que la hipótesis de no degeneración de $D$ sobre $\operatorname{Im} X$ es generalmente satisfecha. En efecto, si $y \in \operatorname{Im} X \cap\left\langle f_{k} / k \in K\right\rangle, y \neq 0$ entonces existen $\alpha_{1}, \ldots, \alpha_{p}$ no todos nulos tales que

$$
y=\sum_{j=1}^{p} \alpha_{j} x^{j}=\sum_{j=1}^{p} \alpha_{j}\left(\sum_{i=1}^{n} x_{i}^{j} f_{i}\right)=\sum_{i=1}^{n}\left(\sum_{j=1}^{p} \alpha_{j} x_{i}^{j}\right) f_{i} .
$$

Se tiene entonces $\sum_{j=1}^{p} \alpha_{j} x_{k}^{j}=0$ para todo $k \notin K$, es decir tal que $p_{k} \neq 0$. Ahora bien, en general $p$ es pequeño respecto al número d'individuos con un peso no nulo, por lo que el sistema de $n-|K|$ ecuaciones con $p$ incógnitas no tiene, en general, solución. 
Puesto que la restricción de $M$ a $\operatorname{Im}{ }^{t} X$ es un producto escalar y teniendo en cuenta las propiedades establecidas para $V M$, los resultados clásicos siguientes [1] sobre los elementos principales del Análisis en Componentes Principales (ACP) del triplete $(X, M, D)$ se enuncian en términos idéntico:

a) Los vectores axiales principales, denotados $c_{1}, \ldots, c_{\text {rang }} X$, forman una base de Im ${ }^{t} X$ constituida por rang $X$ vectores propios $M$-ortonormados de $V M$ asociados a los valores propios no nulos. Para $\lambda_{j}>0$, los vectores $C^{j}=X M c_{j}$ son las componentes principales. Para $\lambda_{j}>0$, se dice que $\left(\lambda_{j}, c_{j}, C^{j}\right)$ es un triplete principal del ACP de $(X, M, D)$.

b) $\mathrm{Si}\left(\lambda_{j}, c_{j}, C^{j}\right)$ es un triplete principal del ACP de $(X, M, D)$, entonces $C^{j}$ es vector propio de $W=X M^{t} X$ asociado al valor propio $\lambda_{j}$.

c) Las componentes principales constituyen una base ortogonal, respecto a $D$, de $\operatorname{Im} X$; es más, $\left\|C^{j}\right\|_{D}=M V M\left(c_{j}, c_{j}\right)=\sqrt{\lambda_{j}}$.

d) Las $C^{j}$ tienen media nula y varianza igual a $\lambda_{j}$.

Como $V M$ es un operador $M$-positivo (c.f. proposición $10(\mathrm{~d})$ ), se tiene que $V M=\sum_{j=1}^{\mathrm{rang} X} \lambda_{j} P_{j}$ (c.f. proposición 14 ); denotando $(V M)^{1 / 2}$ al operador sobre $E$ tal que $(V M)^{1 / 2}=\sum_{j=1}^{\mathrm{rang} X} \sqrt{\lambda_{j}} P_{j}$, se deduce:

- $\operatorname{Im}(V M)^{1 / 2}=\operatorname{Im}\left[\sum_{j=1}^{\operatorname{rang} X} \sqrt{\lambda_{j}} P_{j}\right]=\operatorname{Im}\left[\sum_{j=1}^{\operatorname{rang} X} \lambda_{j} P_{j}\right]=\operatorname{Im} V M=\operatorname{Im}{ }^{t} X$ (c.f. proposición 10(b))

- $\operatorname{ker}(V M)^{1 / 2}=\left(\operatorname{Im}^{t} X\right)^{\perp}=\operatorname{ker} M$ (c.f. $\left.\S 2.1\right)$.

Puesto que $E=\operatorname{Im}^{t} X \oplus \operatorname{ker} M$ (c.f. proposición 4) y $\operatorname{ker}(V M)^{1 / 2}=\operatorname{ker} M$, $(V M)_{/ / \operatorname{Im}{ }^{t} X}^{1 / 2}$ es inyectiva. La inversa generalizada algebraica de $(V M)^{1 / 2}$, denotada $(V M)^{1 / 2-}$, de núcleo $\left(\operatorname{Im}{ }^{t} X\right)^{\perp}$ y de imagen $\operatorname{Im}{ }^{t} X$, es tal que

$$
\forall x \in \operatorname{Im}^{t} X \quad(V M)^{1 / 2-}(x)=(V M)_{/ / \operatorname{Im}}^{-1 / 2}{ }^{t}(x)
$$

donde $(V M)_{/ \operatorname{Im}{ }^{t} X}^{-1 / 2}$ es la aplicación recíproca de $(V M)_{/ \operatorname{Im}{ }^{t} X}^{1 / 2}$ (c.f. proposición 7$)$.

De $(V M)^{1 / 2}=\sum_{j=1}^{\mathrm{rang} X} \sqrt{\lambda_{j}} P_{j}$, se deduce $(V M)_{/ \mathrm{Im}{ }^{t} X}^{-1 / 2}=\sum_{j=1}^{\mathrm{rang} X} \frac{1}{\sqrt{\lambda_{j}}} P_{j}$.

Puesto que $\operatorname{ker}(V M)^{1 / 2-}=\left(\operatorname{Im}{ }^{t} X\right)^{\perp}$ y que $\forall x \in\left(\operatorname{Im}{ }^{t} X\right)^{\perp} P_{j}(x)=O$ (c.f. proposición 14) se deduce de 1: $(V M)^{1 / 2-}=\sum_{j=1}^{\mathrm{rang} X} \frac{1}{\sqrt{\lambda_{j}}} P_{j}$.

Como los $P_{j}$ son $M$-simétricas (proposición 9) y los $\lambda_{j}$ positivos, se tiene el siguiente resultado:

Proposición 21 a) El operador $(V M)^{1 / 2-}$ es $M$-simétrico y $M$-positivo.

b) Si $\left(\lambda_{j}, c_{j}, C^{j}\right)$ es un triplete principal del $A C P$ de $(X, M, D)$, entonces $c_{j}$ es vector propio de $(V M)^{1 / 2-}$ asociado al valor propio $1 / \sqrt{\lambda_{j}}$. 


\section{Demostración:}

a) Mostremos que $(V M)^{1 / 2}$ es $M$-simétrico y $M$-positivo.

Se tiene $\forall(x, y) \in \operatorname{Im}^{t} X \times \operatorname{Im}{ }^{t} X$ :

$M\left[(V M)^{1 / 2} x, y\right]=\sum_{j} \sqrt{\lambda_{j}} M\left(P_{j} x, y\right)=\sum_{j} \sqrt{\lambda_{j}} M\left(x, P_{j} y\right)=M\left[x,(V M)^{1 / 2} y\right]$.

Por lo tanto $(V M)^{1 / 2}$ es $M$-simétrico.

Como $\sqrt{\lambda_{j}}>0$ y los $P_{j}$ son $M$-positivos, se deduce la $M$ positividad de $(V M)^{1 / 2}$.

El final de la demostración es análogo al de la proposición 16.

b) Se tiene $(V M)^{1 / 2} c_{j}=\sum_{k} \sqrt{\lambda_{k}} P_{k} c_{j}=\sqrt{\lambda_{j}} c_{j}$

es más, $c_{j} \in \operatorname{Im}^{t} X$ y $(V M)^{1 / 2-}(V M)^{1 / 2}$ es un proyector sobre $\operatorname{Im}{ }^{t} X$, luego $c_{j}=$ $(V M)^{1 / 2-}(V M)^{1 / 2}\left(c_{j}\right)=\sqrt{\lambda_{j}}(V M)^{1 / 2-}\left(c_{j}\right)$ lo que implica $(V M)^{1 / 2-} c_{j}=\frac{1}{\sqrt{\lambda_{j}}}\left(c_{j}\right)$.

En la proposición que sigue se establecen ciertas propiedades de la aplicación $X M(V M)^{1 / 2-}$, de $E$ en $F$, que serán utilizadas en un próximo artículo para dar expresiones algebraicas a los semiproductos escalares relacionales.

Proposición 22 a) Se tiene $X M(V M)^{1 / 2-}\left(c_{j}\right)=\frac{C^{j}}{\left\|C^{j}\right\|_{D}}$, para todo triplete principal $\left(\lambda_{j}, c_{j}, C^{j}\right) \operatorname{del} A C P$ de $(X, M, D)$.

b) $\operatorname{Im}\left[X M(V M)^{1 / 2-}\right]=\operatorname{Im} X$.

c) $\operatorname{ker}\left[X M(V M)^{1 / 2-}\right]=\left(\operatorname{Im}{ }^{t} X\right)^{\perp}$.

\section{Demostración:}

a) Se tiene $(V M)^{1 / 2-}\left(c_{j}\right)=\frac{1}{\sqrt{\lambda_{j}}} c_{j}$ (c.f. proposición 21(b)). Ahora bien, $\sqrt{\lambda_{j}}=\left\|C^{j}\right\|_{D}$, de donde $(V M)^{1 / 2-} c_{j}=\frac{c_{j}}{\left\|C^{j}\right\|_{D}}$. Por lo tanto, se obtiene $X M(V M)^{1 / 2-}\left(c_{j}\right)=$ $X M\left(\frac{c_{j}}{\left\|C^{j}\right\|_{D}}\right)=\frac{C^{j}}{\left\|C^{j}\right\|_{D}}$.

b) Se deduce inmediatamente de (a).

c) Se tiene $\operatorname{ker} X M(V M)^{1 / 2-} \supset \operatorname{ker}(V M)^{1 / 2-}=\operatorname{ker} M . \quad$ Es más, $X M(V M)^{1 / 2-}\left(c_{j}\right)=\frac{C^{j}}{\left\|C^{j}\right\|_{D}}, \operatorname{luego} \operatorname{dim}\left[\operatorname{Im} X M(V M)^{1 / 2-}\right]=\operatorname{dim}(\operatorname{Im} X)=\operatorname{rang} X$ (c.f. (c) de la page 51). Ahora bien, $E=\operatorname{Im}^{t} X \oplus \operatorname{ker} M$, entonces $\operatorname{dim}(\operatorname{ker} M)=\operatorname{dim} E-\operatorname{rang} X=\operatorname{dim}\left[\operatorname{ker} X M(V M)^{1 / 2-}\right] . \quad$ Por lo tanto $\operatorname{ker} X M(V M)^{1 / 2-}=\operatorname{ker} M=\left(\operatorname{Im}^{t} X\right)^{\perp}$.

Obsérvese que $X M(V M)^{1 / 2-}$ es una isometría de $\operatorname{Im}{ }^{t} X$ en $\operatorname{Im} X$.

\section{Conclusiones y perspectivas}

Se han establecido propiedades útiles de los semiproductos escalares, que han servido para trabajar en una definición de las semimétricas útiles en Análisis de Datos. Tanto en el 
espacio de individuos como en el espacio de variables, el uso de estas semimétricas ha permitido trabajar de manera que se puede desarrollar una teoría coherente.

En una próxima publicación, extenderemos estas ideas al caso de semimétricas relacionales, es decir, definidas por bloques, las cuales serán útiles para el caso de trabajar con tablas múltiples o bien para extender una serie de técnicas de Análisis de Datos.

\section{Referencias}

[1] Cailliez, F.; Pagès, J.P. (1976) Introduction à l'Analyse des Données. SMASH, Paris

[2] Escofier, B.; Pagès, J. (1988) Analyses Factorielles Simples et Multiples. Dunod, Paris.

[3] Glazman, I.; Liubitch, Y. (1972) Analyse Linéaire dans les Espaces de Dimensions Finies. Editions MIR, Moscou.

[4] Grifone, J. (1990) Algèbre Linéaire. Cépaduès-Editions, Toulouse.

[5] Halmos, P.R. (1958) Finite Dimensional Vector-Spaces. Van Nostrand, Princeton, New Jersey.

[6] Nashed, Z. (ed.) (1976) Generalized Inverses and its Applications. Academic Press Inc., New York.

[7] Pontier, J.; Dufour, A.-B; Normand, M. (1991) Le Modèle Euclidien en Analyse des Données. S.M.A., Editions Ellipses, Bruxelles.

[8] Queysanne, A. (1964) Algèbre. Armand Colin, Ed., Paris.

[9] Schektman, Y. (1978) Contribution à la Mesure en Facteurs dans les Sciences Expérimentales y à la mise en Euvre Automatique dans les Calculs Statistiques. Thèse d'Etat, Toulouse.

[10] Schektman, Y. (1988) Analyse et Traitement Informatique de Données. Cours de D.E.A., Université Paul Sabatier, Toulouse.

[11] Schektman, Y. (1991) "Eléments mathématiques de base pour la définition de semiproduits scalaires utiles en analyse de données. Quelques applications", Note interne, Laboratoire MLAD, Université Paul Sabatier, Toulouse.

[12] Schektman, Y.; Troupé, M.; Trejos, J. (1992) "Un générateur de règles floues p̀artir de bases de données volumineuses", In: E. Diday \& Y. Kodratoff (Eds.) III Journées Symboliques-Numériques, Université Paris IX-Dauphine, pp. 121-130.

[13] Troupé, M. (1994) Contribution à la Protection de la Régression Multiple MMultidimensionnelle et la Génération de Règles Prévisionelles. Thèse de Doctorat, Université Paul Sabatier, Toulouse.

[14] Trejos, J. (1994) Contribution à l'Acquisition Automatique de Connaissances à Partir de Données Qualitatives. Thèse de Doctorat, Université Paul Sabatier, Toulouse. 